\title{
Comments on 'Hebbian learning is jointly controlled by electrotonic and input structure'
}

\author{
Barak A Pearlmutter $\dagger$ \\ Siemens Corporate Research, 755 College Road East, Princeton, NJ 08540, USA
}

Received 6 June 1994

\begin{abstract}
It is argued that simulations presented by Tsai, Carnevale and Brown do not agree with their theoretical predictions and that their mathematical derivation contains a major flaw. The origin of these misunderstandings is traced to the application of a special case of an equation whose general version is given here.
\end{abstract}

I would like to correct a number of errors that crept into Tsai, Carnevale and Brown (1994). Equation (10) and the remainder of the paper do not follow from equation (9). Equation (9) retains the decay term from Oja's rule (1982) in the non-isopotential situation, where it loses its desirable mathematical properties, making equation (10) incorrect. If the weights are to converge to the principal eigenvector of $\mathbf{C}$, a decay term like that of Yuille et al (1989) must be used (Miller and MacKay 1994, Goodhill and Barrow 1994). This accounts for the failure to use equation (10) in a predictive fashion, for in the simulations shown the weights do not converge to the principal eigenvector of $\mathbf{C}$.

In remarks following equation (12), it is stated that this decoupled form of the equation supplies 'less useful neurophysiological insight' than equation (10). Fortunately the insights to be had are computational, not neurophysiological. When it holds, equation (12) shows that the input's only important property is its pairwise correlational structure. Equation (12) is also interesting because of its potential applications. Just as Oja's equation for the instantaneous isopotential case was used to account for receptive fields in the visual system (Miller et al 1989), this equation can extend such analysis to non-isopotential neurons. And just as Chernjayski and Moody (1990) used an identical equation to predict the length scale of cortical columns, one might predict the length scale of clusters of synaptic facilitation along a dendritic shaft.

The last paragraph on page 8 says that the instant at which Hebbian modulation occurs is both simultaneous with the instantaneous presynaptic activity and subsequent to the presynaptic activity's postsynaptic effect. This would have delighted Bishop Berkeley (1734). Equation (9) is a special case of

$$
\frac{\mathrm{d} m_{i}}{\mathrm{~d} t}=\eta \int_{0}^{\infty} \xi_{i}(t-\tau) \psi_{i}(\tau) \mathrm{d} \tau v_{i}(t)-\text { decay }
$$

where $v_{i}(t)=\sum_{j} w_{j} \int_{0}^{\infty} \xi_{j}(t-\tau) \tilde{v}_{i j}(\tau) \mathrm{d} \tau$ is the postsynaptic potential, $\xi_{i}(t)$ is the input to synapse $i$ at time $t, \tilde{v}_{i j}(\tau)$ is the neuron's voltage response to a unit current injection at

† E-mail: bap@learning.scr.siemens.com 
synapse $j$ as measured $\tau$ seconds later at synapse $i$, and we introduce $\psi_{i}(\tau)$ as the time course of opportunity for modulation of synapse $i$ following presynaptic activity. This leads to

$$
\langle\mathrm{d} m / \mathrm{d} t\rangle=\eta \mathrm{C} m-\langle\text { decay }\rangle
$$

in which $\mathbf{C}$ is defined by the more general

$$
C_{i j}=\int_{0}^{\infty} \tilde{v}_{i j}(\tau)\left(Q_{i j} * \psi_{i}\right)(\tau) \mathrm{d} \tau
$$

where $Q_{i j}(\tau)=\left\langle\xi_{i}(t) \xi_{j}(t-\tau)\right\rangle$ (Pearlmutter 1994). To get the special case of Pearlmutter and Brown (1992), one considers $\psi(\tau)$ to be a Dirac delta pulse and takes the limit as this pulse is moved towards $0+$.

Brown, Carnevale and Tsai had access to a preliminary draft of Pearlmutter (1994). That mansucript, and the short abstract Pearlmutter and Brown (1992), suffered from an overly terse mathematical formulation. The more leisurely exposition in the final publication should reduce the possibility of this sort of confusion.

\section{References}

Berkeley G 1734 The Analyst: A Discourse Addressed to an Infidel Mathematician, Wherein it is Examined Whether the Object, Principles and Inferences of the Modern Analysis are More Distinctly Conceived, or More Evidently Deduced, than Religious Mysteries and Points of Faith (reprinted in: Fraser A C (ed) 1901 Berkeley's Complete Works (Oxford: Clarendon Press))

Chernjavski A and Moody J E 1990 Spontaneous development of modularity in simple cortical models Neural Computation 2 (3) 334-54

Goodhill G J and Barrow H G 1994 The role of weight normalization in competitive learning Neural Computation 6 (2) 255-69

Miller K D, Keller J B and Stryker M P 1989 Ocular dominance column development: analysis and simulation Science 245 605-15

Miller K D and MacKay D J C 1994 The role of constraints in Hebbian leaming Neural Computation 6 (1) 100-126

Oja E 1982 A simplified neuron model as a principal component analyzer J. Math. Biol. 15 267-73

Pearlmutter B A 1992 Time-skew Hebb rule in a non-isopotential neuron Neural Computation submitted

Pearlmutter B A and Brown T H 1992 Hebbian learning is jointly controlled by electrotonic and input structure Soc. Neurosct. Abstracts 18 (567.23)

Tsai K Y, Camevale N T and Brown T H 1994 Hebbian learning is jointly controlled by electrotonic and input structure Network 5 (1) 1-19

Yuille A L, Kammen D M and Cohen D S 1989 Quadrature and the development of orientation selective cortical cells by Hebb rules Biol. Cybern. 61 183-94 